\title{
Linx
}

Revue des linguistes de l'université Paris X Nanterre

$8 \mid 1996$

Du dire et du discours

\section{Variations sur langage et métalangage}

\section{Marie-Françoise Mortureux}

\section{(2) OpenEdition \\ Journals}

Édition électronique

URL : http://journals.openedition.org/linx/1094

DOI : 10.4000/linx.1094

ISSN : 2118-9692

\section{Éditeur}

Presses universitaires de Paris Nanterre

\section{Édition imprimée}

Date de publication : 1 septembre 1996

Pagination : $3-12$

ISSN : 0246-8743

\section{Référence électronique}

Marie-Françoise Mortureux, "Variations sur langage et métalangage », Linx [En ligne], 8 | 1996, mis en ligne le 11 juillet 2012, consulté le 19 avril 2019. URL : http://journals.openedition.org/linx/1094 ; DOI : 10.4000/linx.1094

Ce document a été généré automatiquement le 19 avril 2019.

Département de Sciences du langage, Université Paris Ouest 


\title{
Variations sur langage et métalangage
}

\author{
Marie-Françoise Mortureux
}

1 Depuis que R. Jakobson, puis E. Benveniste ont souligné, l'un à travers la fonction métalinguistique, l'autre à travers la double signifiance de la langue, cette capacité particulière des langues naturelles de parler d'elles-mêmes aussi bien que du monde, les linguistes se sont attachés à explorer les réalisations diverses de cette compétence, dont rend compte l'opposition entre métalangage et langage mondain. Mais l'attention portée à ce phénomène a fait émerger son originalité singulière, qui tient à ce que cette double signifiance peut se réaliser simultanément sur une même séquence linguistique : discours métalinguistique et discours mondain se superposent, en se doublant l'un l'autre, (ce que désigne notamment le concept de fonction, utilisé par Jakobson, car les fonctions ne sont pas exclusives les unes des autres.).

2 Quelques exemples seront ici présentés, de discours entremêlant métalangage et sémiotique mondaine, qui permettront de préciser la notion de représentation de la langue, dans ses rapports avec le discours sur la langue et la mise en discours de la langue. Ces exemples proviennent de travaux conduits ces dernières années avec des étudiants de licence en Sciences du langage à l'Université de Nanterre.

\section{Exemples dans le dictionnaire de langue}

3 La sémiotique lexicographique est bien connue pour faire co-fonctionner des éléments franchement métalinguistiques (telles les gloses définitionnelles ou les indications reliant les diverses acceptions proposées pour un même lexème) et des fragments, lambeaux empruntés aux discours mondains, ce qu'on appelle généralement les exemples. Parmi eux figurent (selon les ouvrages) des citations de textes écrits, le plus souvent littéraires.

4 L'observation des rapports entre les dictionnaires (de langue) et la littérature est l'un des thèmes fréquemment traités par la métalexicographie. Il a inspiré récemment un colloque qui s'est tenu à Lille en $1991^{1}$, et dont les Actes sont en cours de publication ${ }^{2}$. En règle générale, ce qui retient l'attention des métalexicographes, c'est le rôle que joue 
dans le discours lexicographique le recours à la citation littéraire, dans une perspective autant (sinon plus) idéologique (quelle image des usages, des stéréotypes socioculturels véhicule le dictionnaire?) que (méta)linguistique (quelle information sur la langue et sur le modèle d'analyse mis en oeuvre apporte l'exemple?).

Cette dernière question sera ici abordée à partir des réactions d'étudiants confrontés au discours lexicographique. Invités à comparer dans deux dictionnaires de langue contemporains et de dimension analogue (le Petit Robert et le Lexis) la représentation du champ des bateaux (bateau, barque, bâtiment, canot, cargo, embarcation, navire, paquebot, vaisseau, vedette), certains d'entre eux avaient vivement critiqué la citation des Mémoires du Général de Gaulle dans l'article cargo du Lexis : «Je fis retenir ma place à bord d'un cargo français qui s'apprêtait à gagner le Maroc (de Gaulle).»

6 Cet exemple, en effet, leur semblait contredire la définition proposée: «Navire spécialement destiné au transport des marchandises».

7 La difficulté soulevée par cette remarque peut être examinée de deux points de vue :

- en partant de la citation littéraire; alors on peut soutenir que l'expressivité de la phrase citée s'appuierait effectivement sur l'information touchant l'affectation du cargo au transport de marchandise ; cette expressivité tiendrait à la particularité de ce voyage, due, selon les cas, à l'urgence de la situation ;

- en partant de la paraphrase définitionnelle, et, d'une façon générale, du modèle sémantique que révèle la comparaison des deux dictionnaires. Cette comparaison suggère que chacun d'eux adopte une stratégie différente. Sans reproduire ici les détails de l'analyse, on peut dire ceci :

- le Lexis procède, semble-t-il, par oppositions. En effet, l'examen de l'ensemble des paraphrases définitionnelles proposées pour les mots-entrées mentionnés ci-dessus fait apparaître un système reposant sur la distinctivité et la hiérarchie, conformément au schéma de la sémantique componentielle ${ }^{3}$. Dans cette structure représentable en arbre ${ }^{4}$, cargo, dominé par navire, est co-hyponyme de paquebot; l'opposition des traits (marchandises/personnes) s'impose tout naturellement. Et c'est la définition de cargo mixte qui tend à neutraliser cette opposition à travers la glose développée suivante: «cargo aménagé pour recevoir un petit nombre de passagers, en sus de sa cargaison.» La sémantique qui inspire cet ouvrage serait celle des CNS (conditions nécessaires et suffisantes) dont on sait qu'elle est régulièrement prise en défaut par la diversité des emplois en discours, dont beaucoup n'actualisent pas la totalité des traits retenus par une telle analyse sémantique ${ }^{5}$. Ce qui explique sans doute l'ampleur de la glose définitionnelle de cargo mixte, destinée aussi à légitimer la présence, dans le discours lexicographique immédiatement précédent, de l'exemple emprunté à de Gaulle. En revanche, la sémantique des CNS donne du lexique une image plus conforme au modèle structural, et souligne la différence entre système linguistique (abstrait) et réalisations discursives, entre lexème et vocable ${ }^{6}$.

- Le Petit Robert ne dessine pas un schéma aussi net de ce champ. Il propose comme définition de cargo : «Navire destiné surtout ${ }^{7}$ au transport des marchandises», substituant l'adverbe surtout à spécialement qui figure dans le Lexis; cette substitution, qui prévient la contradiction, et contourne la difficulté, relève d'une logique dite aujourd'hui prototypique . $^{8}$ Cela lui permet de mentionner dans le même article le syntagme cargo mixte, avec une définition brève : «qui peut prendre des passagers». La même citation de de Gaulle, importée dans le Petit Robert, y perdrait toute valeur expressive pour n'y être qu'un exemple d'emploi banal, courant, comme l'attendaient en fait les étudiants. 
Le jeu de la citation apparaît alors, à travers cet exemple, totalement pris dans le discours lexicographique déterminé par le modèle sémantique de référence, et non réductible à une simple connotation culturelle, idéologique. Son interprétation joue simultanément sur les deux registres: dans l'ordre du métalangage, il signifie l'écart entre emploi prototypique et emploi non prototypique; dans l'ordre mondain, la valeur de l'information (expressive ou non) en est affectée.

\section{Article de dictionnaire converti en récit}

Dans Les fleurs bleues, Queneau écrit :

« [Le duc d'Auge] quitta son poste de guet pour les étages inférieurs du château en se livrant au passage à son humeur qui était de battre.

Il ne battit point sa femme parce que défunte, mais il battit ses filles au nombre de trois; il battit des serviteurs, des servantes, des tapis, quelques fers encore chauds, la campagne, monnaie et, en fin de compte, ses flancs. $»^{9}$

10 Formellement, cette dernière phrase s'analyse très clairement comme la projection sur l'axe syntagmatique d'une partie du paradigme des emplois de battre, dans l'ordre où ils sont présentés par un dictionnaire comme le Petit Robert - à une exception près : dans l'article de dictionnaire, battre monnaie précède battre la campagne. ${ }^{10} \mathrm{La}$ mise en discours de ces extraits d'article de dictionnaire s'accompagne de quelques manipulations lexicosyntaxiques : calembour sur le second emploi (battre quelqu'un [qui est] à SN / battre quelqu'un à SN (où $\mathrm{N}$ désigne une compétition : jeu, sport, politique,...)); défigement de battre le fer tant qu'il est chaud et de se battre les flancs. Ces opérations soulignent le caractère ludique d'un texte qui apparait d'abord comme un pur «jeu de mots», du métalangage dépourvu de référence mondaine.

11 Cependant, comme l'affirment les étudiants, passé le premier éclat de rire, « on voit bien ce que ça veut dire : le duc est furieux ». Le sémantisme initial du verbe origine, comme le rythme de la phrase, déjà évoqué, y sont pour beaucoup. Mais cette information globale ne suffit pas à épuiser le sens du texte. En effet, mise à part l'occurrence de monnaie, cet énoncé se laisse décomposer en deux grandes parties: la première décrit des actions physiques sur des êtres humains ou des tapis, tandis que la seconde fait intervenir des syntagmes figés, dotés en langue d'une interprétation dite "figurée ${ }^{11}$; celle-ci s'explicite successivement par: "profiter sans tarder d'une occasion favorable, rêver à des sujets variés (sans ordre), faire des efforts inutiles» (Petit Robert); l'agitation physique initiale se trouve réinterprétée comme la manifestation d'une agitation psychique qui se résout ainsi dans la phrase suivante : «Tout de suite après, il décida de faire un court voyage...» (p. 14). Le duc, héros de l'histoire, passe alors de la contemplation qui ouvre le livre à l'action dont le récit occupe ce livre avant de se refermer, dans les dernières lignes, sur le retour à la contemplation. Il y a donc bien réinvestissement de la représentation de la langue dans un discours qui n'est pas pur métalangage, mais est doté - secondairement ? - d'une référence mondaine, fictive, certes, mais néanmoins identique à celle des textes «littéraires» en général. La connotation autonymique, définie par J. Rey-Debove comme caractéristique de la littérature, semble franchir un niveau supplémentaire ici : en effet, le mode du «comme je dis » se trouve redoublé d'un mode du « comme on dit ».

Dans ce dernier texte, comme dans le cas précédent (citation lexicographique), la représentation de la langue (là, la signification du nom cargo,ici, le paradigme des 
constructions du verbe battre) s'impose à l'interprétation au point de mettre en cause (pour le premier) ou de reléguer d'abord au second plan (pour le second) le sens mondain de la phrase dans le texte dont elle est extraite. Ce serait l'inverse dans d'autres textes ludiques également, comme les sketches de R. Devos.

\section{Polysémie, synonymie : silence !}

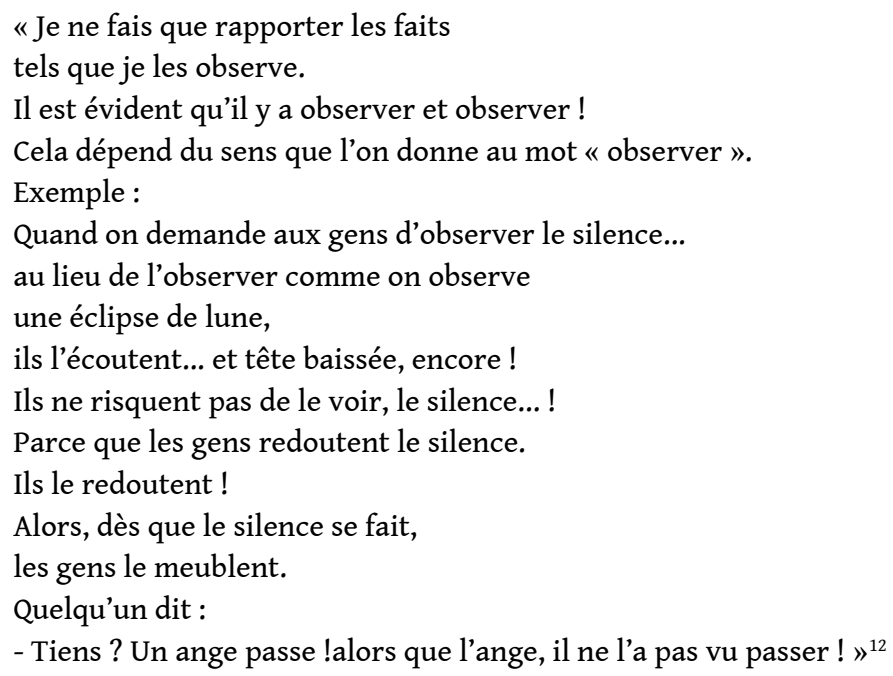

L'exemplification de la polysémie de observer, que prétend proposer ici R. Devos, prend la forme d'une historiette qui se poursuit à travers l'occurrence des verbes écouter, voir, redouter, meubler. Chacun de ces cinq verbes y figure avec pour complément le silence. Et, pour finir, le comédien s'adressera ainsi à son public :

« Mesdames et messieurs... à la mémoire de tous les anges

qui sont tombés dans cette salle,

nous allons observer une minute de silence... »

14 C'est donc en fait le paradigme des verbes formant avec ce nom silence une expression sinon figée, du moins stéréotypée ${ }^{13}$, que déroule ce texte. Le déroulement de ce paradigme, et son exhibition, passe par l'occurrence des verbes, eux-mêmes polysémiques, écouter et voir, employés comme « verbes de perception » ${ }^{14}$. Naturellement, l'exemple proposé constitue une démonstration par l'absurde, puisque le verbe observer n'a pas la même acception dans la première occurrence ("les faits tels que je les observe») et les suivantes ("observer le silence»). Cette "erreur» manifeste la polysémie, que fait fonctionner la démonstration, la mettant en discours en réalisant plusieurs des «synonymies locales» qu'autorisent les commutations appropriées. Ce procédé très répandu, en quoi consiste maint jeu de mots, exploite, certes, comme n'importe quel discours mondain, la structure lexicale, le réseau tissé par la polysémie et la synonymie, mais - ce que ne font pas la plupart des discours mondains, les discours " ordinaires ", c'est-à-dire non marqués à cet égard - il attire l'attention du récepteur sur ce réseau, en faisant de son fonctionnement discursif le principe même du discours.

Les extraits de discours précédents avaient en commun d'être générés par un paradigme linguistique parfaitement défini; ils pouvaient apparaitre comme la conversion discursive d'un fragment de dictionnaire (constructions de battre, acceptions d'observer, locutions verbales formées avec silence comme complément). Ce n'est pas le cas du 
dernier exemple que l'on veut évoquer ici, lequel enchaîne des expressions reliées par diverses associations.

\section{Associations}

«[...] si l'on savait ce qui amuse les atomes, on leur fournirait matière à rire[...] si

bien qu'on ne les ferait plus éclater que de rire.

Alors, me direz-vous, que deviendrait la fission nucléaire?

Une explosion de joie !»

Le jeu de la double isotopie repérable ici à travers l'occurrence de atomes, matière, éclater, fission nucléaire, explosion d'une part, et amuse, matière à rire, éclater de rire, explosion de joie de l'autre, repose sur ces trois dernières expressions, toutes trois parfaitement stéréotypées. Le premier élément de deux d'entre elles (matière (à rire), explosion (de joie)) appartient au champ sémantique de l'atome, et de la bombe atomique; le verbe éclater est mentionné comme analogue de exploser, et vice-versa dans le Petit Robert ; la relation entre fission nucléaire et explosion est indirectement indiquée dans ce dictionnaire, à travers la mention de la bombe dans les articles fission et exploser. Il ne s'agit même plus ici de polysémie explicite, mais simplement de la diversité d'emploi syntagmatique de lexèmes dont la signification de base ne varie guère; du moins si l'on admet la régularité des emplois métaphoriques (dits "figurés») du type éclater/éclater de rire, c'est-à-dire la double valeur (physique/psychique) de maint verbe ou nom. Dans ce sketch, l'interprétation mondaine (comment échapper à la terreur nucléaire) s'impose, certes, avant tout ; cependant, les jeux de mots, s'ils constituent la matière verbale du propos, n'en soulignent pas moins le tissu associatif dont est fait le lexique.

Ces quatre exemples ont en commun d'illustrer le fonctionnement de la double signifiance de la langue. Ils s'échelonnent du plus explicitement métalinguistique (la citation littéraire dans un dictionnaire de langue) au plus discrètement épilinguistique. Ils évoquent tour à tour la sémantique lexicale, la syntagmatique verbale, et la structure morphosémantique du lexique.

Loin d'être une rareté, les discours susceptibles d'illustrer cette propriété des langues naturelles sont très répandus. Le moindre slogan publicitaire peut exhiber des effets phoniques ou lexicaux, voire syntaxiques, pour augmenter l'impact de son message. A l'autre extrémité de cette gamme, figurent les exemples, et contre-exemples, des études linguistiques contemporaines: leur efficacité et leur pertinence reposent sur leur aptitude à être reconnus comme des discours produits (et attestables...) dans la langue que l'on cherche à décrire, alors même que leur fonction dans le discours du linguiste est métalinguistique.

Si la langue ne s'observe que dans le discours, la variété des performances envisagées sous l'angle sémiotique (métalangage/langage mondain) offre une gamme très riche et finement échelonnée de discours superposant mise en fonctionnement et mise en scène de la langue. Rares, sans doute, sont les discours "purs », purement métalinguistiques (études linguistiques en langue naturelle, non formalisées, et dépourvues de tout exemple) ; à l'inverse, toute mise en fonctionnement de la langue dans la communication n'engage-t-elle pas une (dé)monstration du système, qu'exploite précisément toute étude linguistique empirique (s'appuyant sur un corpus) ? L'opposition métalangage/langage mondain induit donc une interprétation (clivée, dédoublée) des segments linguistiques, et, plus que leur bipartition, leur échelonnement sur un axe en fonction de leur « densité 
métalinguistique ». Ce concept, utilisé par J. Rey-Debove dans sa description des phrases métalinguistiques, permet en effet de rendre compte des innombrables réalisations qui mettent en jeu la compétence métalinguistique des locuteurs: des prédications proprement métalinguistiques observer est polysémique, ou encore observer a deux significations, qui ont pour thème un autonyme, et dont le propos contient des termes métalinguistiques (polysémique, signification), à la remarque largement mondaine il y a observer et observer, où peut ne figurer ni l'un ni l'autre ${ }^{15}$, en passant par un intermédiaire comme "cela dépend du sens que l'on donne au mot 'observer'", où le terme métalinguistique mot et l'autonyme observer ne figurent que dans le propos, on parcourt la gamme du plus au moins dense. Particulièrement adaptée aux cas intermédiaires ou douteux, l'observation de la densité métalinguistique permet, plus que tout autre approche, de décrire le fonctionnement discursif de la double signifiance linguistique.

On voit ainsi se développer une démarche qui, s'éloignant d'une logique du tiers exclu, met en oeuvre une description plus fine, dans laquelle les termes définis précédemment en opposition jouent le rôle de bornes aux extrémités d'une échelle. Evolution qui s'observe ailleurs, notamment en sciences humaines.

\section{BIBLIOGRAPHIE}

BENVENISTE, E. (1966 et 1974) Sémiologie de la langue, Problèmes de linguistique générale, 2 tomes, Paris, Gallimard.

JAKOBSON, R. (1963) Essais de Linguistique générale, Paris, éd. de Minuit.

KLEIBER, G. (1990) La sémantique du prototype, Paris, PUF.

REY-DEBOVE, J. (1978) Le métalangage, Paris, éd. Le Robert.

\section{NOTES}

1. Dictionnaire et littérature 1830-1990, colloque organisé par l'URA/SILEX 382 et le Centre d'Analyse et de Critique des textes de l'Université Lille III, 1991.

2. Voir Langue française 106 et Lexique 12-13.

3. Ainsi: bateau "terme générique désignant toute sorte de navire ou d'embarcation». embarcation "terme générique pour désigner tous les petits bateaux». navire "bateau, en général de fort tonnage ». cargo «navire spécialement destiné au transport des marchandises». paquebot « grand navire destiné au transport des personnes ».

4. Le système n'est pas parfait, toutefois : la distinctivité s'estompe entre barque et embarcation, d'une part; et les relations entre le couple cargo/paquebot d'une part et les unités bâtiment, vaisseau de l'autre sont floues. Globalement, cependant, il s'agit bien d'une structure clairement hiérarchisée.

5. La phrase de de Gaulle estompe la valeur de l'adverbe "spécialement ", et, d'une certaine façon, cargo y fonctionne comme équivalent abrégé de cargo mixte. 
6. Les deux termes sont eux-mêmes définis ainsi dans l'ouvrage : lexème « morphème lexical ( table, roi, venir, etc.) par opposition au morphème grammatical (de, le, avec, etc.) »; vocable « mot, terme désignant un objet, une notion, etc. syn. dénomination ».

7. C'est moi qui souligne l'adverbe.

8. Le principe d'une démarche prototypique consiste à distinguer un noyau (prototype) et une périphérie. Pour une catégorie donnée (exemple l'oiseau), on considère que certains exemplaires sont plus représentatifs que d'autres: ainsi le moineau peut apparaître comme le meilleur exemplaire d'oiseau, l'autruche comme l'un des moins bons. Mais le nom d'oiseau s'appliquera cependant aux deux. Appliquée aux définitions lexicogaphiques, l'approche prototypique conduit notamment, comme ici, à éviter les oppositions qui seraient prises en défaut par de trop nombreux usages. Sur le prototype, et son application au lexique, voir les travaux de G. Kleiber, cités en bibliographie.

9. Les fleurs bleues, folio, éd. Gallimard, Paris, p.14.

10. Le rythme de la phrase de Queneau, qui contribue à connoter une agitation aussi violente que vaine, peut expliquer cette particularité.

11. A cet égard aussi, la place de l'occurrence de monnaie apparaît anomale.

12. Un ange passe, Matière à rire, p. 99.

13. Un syntagme figé s'interprète conventionnellement, sa signification est globalement arbitrarisée; dans le cas du sketch, les expressions utilisées peuvent s'interpréter à partir de la signification des unités lexicales qui les composent... à condition, évidemment, de sélectionner convenablement l'acception du verbe qui est concernée. Du reste, aucune de ces expressions n'était répertoriée dans le Petit Robert en 1979.

14. Les deux verbes sont, certes, hyponymes de percevoir; mais ils ne sont pas cohyponymes : en effet, si voir c'est "percevoir par les yeux ", écouter c'est "s'appliquer à entendre ", ce dernier verbe étant, comme verbe de perception, cohyponyme de voir.

15. Observer peut, semble-t-il, s'interpréter ici de façon purement mondaine : «il y a plusieurs façons d'observer, il y a plusieurs sortes d'observation(s) ».

\section{RÉSUMÉS}

Bien des énoncés supportent une double interprétation, mondaine et métalinguistique, intriquées. L'opposition entre métalangage et langage mondain conduit donc moins à une bipartition des discours qu'à leur échelonnement en fonction de leur densité métalinguistique.

A double interpretation, metalinguistic and ordinary, insetled, applies to many linguistic sequences. From the opposition between metalanguage and ordinary language, we can investigate some discourse's classification, based on metalinguistic density, and not bipartition. 
AUTEUR

MARIE-FRANÇOISE MORTUREUX

Professeur émérite à l'Université de Paris X

17 rue du colonel Oudot 75012 PARIS 